\title{
Analysis of Transit Network Design Using GIS and Honeybee Algorithm in the City of Sanandaj, Iran
}

\author{
Armin Ahmadi \\ Geography Department, Western University, London, Canada \\ Email: aahma2@uwo.ca
}

How to cite this paper: Ahmadi, A. (2020) Analysis of Transit Network Design Using GIS and Honeybee Algorithm in the City of Sanandaj, Iran. Journal of Geographic Information System, 12, 716-731.

https://doi.org/10.4236/igis.2020.126041

Received: October 9, 2020

Accepted: December 18, 2020

Published: December 21, 2020

Copyright $\odot 2020$ by author(s) and Scientific Research Publishing Inc. This work is licensed under the Creative Commons Attribution International License (CC BY 4.0).

http://creativecommons.org/licenses/by/4.0/ (c) (i) Open Access

\begin{abstract}
Public transit planning is a user-oriented problem, respectful of financial issues and involves different stakeholders such as the general public, the transportation provider and the local government. One of the main components of public transit planning is the transit network design (TND) problem. This research is an attempt to perform transit network design and analysis in the city of Sanandaj, Iran using the capabilities of GIS and Honeybee algorithm. Objectives of this study are formulating a multi-objective model of the TND problem, developing a GIS-based procedure for solving the TND problem and examination of the solutions using artificial metaheuristic methods such as honeybee algorithm. The transit network design approach in this research, aims to reduce the walking distance, the total travel distance and the total number of stops needed for a suitable transit service in Sanandaj, Iran. One of the contributions of this research is developing a transit network design with utilizing a spectrum of GIS software modelling functionalities and using the abilities of the artificial intelligence in modelling and assessment of the transit network.
\end{abstract}

\section{Keywords}

Transit Network Design (TND), GIS, Honeybee Algorithm, Coverage, Planning

\section{Introduction}

Public transit system in Sanandaj has been under review and modification for several years to reduce the traffic congestion and the share of private car usage in the city and increase the very low share of public transit [1]. The bus routes in 
Sanandaj are not connected to each other and link the downtown core to the peripheries without providing travel options for passengers between peripheries. The lack of service in many populated districts of Sanandaj and town nearby makes the transit system unpopular and unreliable.

Transit network design is a strategic planning problem aiming at maximizing service quality under budgetary restrictions. It is a method of finding a set of routes with specific schedules for a transit fleet [2]. Because of the large number of the bus stops, it is difficult to find a set of routes using the traditional approaches [3]. There are two main approaches to TND: the route level design and the network level design [4]. Route level design focuses on a small part of the network or a single transit route to manage new travel demand and reduce the number of transfers and circuity. The network approach is to design the transit network as a set of routes to serve the current travel demand efficiently.

Accessibility for the population with limited access to the private transportation is a major objective of the public transit system [5]. Accessibility is "the ease with which activities at one place may be reached from another via a particular travel mode" [6]. Spatial accessibility includes access and geographic coverage (Murray \& Wu, 2003). Lower walking distance from the transit network to the attractive destinations, such as shopping malls, or population centers generates a higher level of accessibility (S. Liu \& Zhu, 2004). GIS will help to find the level of access and coverage of the designed transit network alternatives based on the distance to population centers and attractive locations.

The main goal of this research is to develop a multi-objective GIS-based procedure for the TND problem and apply the proposed approach to construct and to evaluate several bus network alternatives in Sanandaj. For the stated goal, the following objectives of this study are set to achieve:

1) To formulate a multi-objective model of the TND problem.

2) To develop a GIS-based procedure for solving the TND problem.

3) To examine the solutions (alternative transit networks) using metaheuristic methods such as honeybee algorithm.

\section{Study Area}

Kurdistan province with an area of 28,203 square kilometer which makes up 1.7 percent of Iran's land. The province consists of plains and valleys in Zagros mountains. To the north, the province is neighboring Azarbaijan, to the east Hamedan and Zanjan, and to the south Kermanshah provinces. Kurdistan province is also on the Iraqi border, it is connected to Iraq from West.

Sanandaj is the capital of the Kurdistan province. The city's population was 432,330 in 2011 census [1]. Sanandaj distance from Tehran (capital city) is 520 kilometers. Figure 1 displays the street network in Sanandaj. Figure 2 also displays the roads and several popular destinations in Sanandaj that people visit, work or shop in those locations during the day. These destinations include hospitals, parks, movies theaters, Bazaar, government offices, retail market, indus- 
trial sites and other popular locations in the city. Due to lack of efficient bus service only two percent of the trips to and from these locations are using the current bus system in Sanandaj (Figure 3). Therefore, there is an urgent need to redesign the bus network in Sanandaj in order to provide a better service for the population centers and attractive destinations and reduce the traffic congestion in Sanandaj [1].

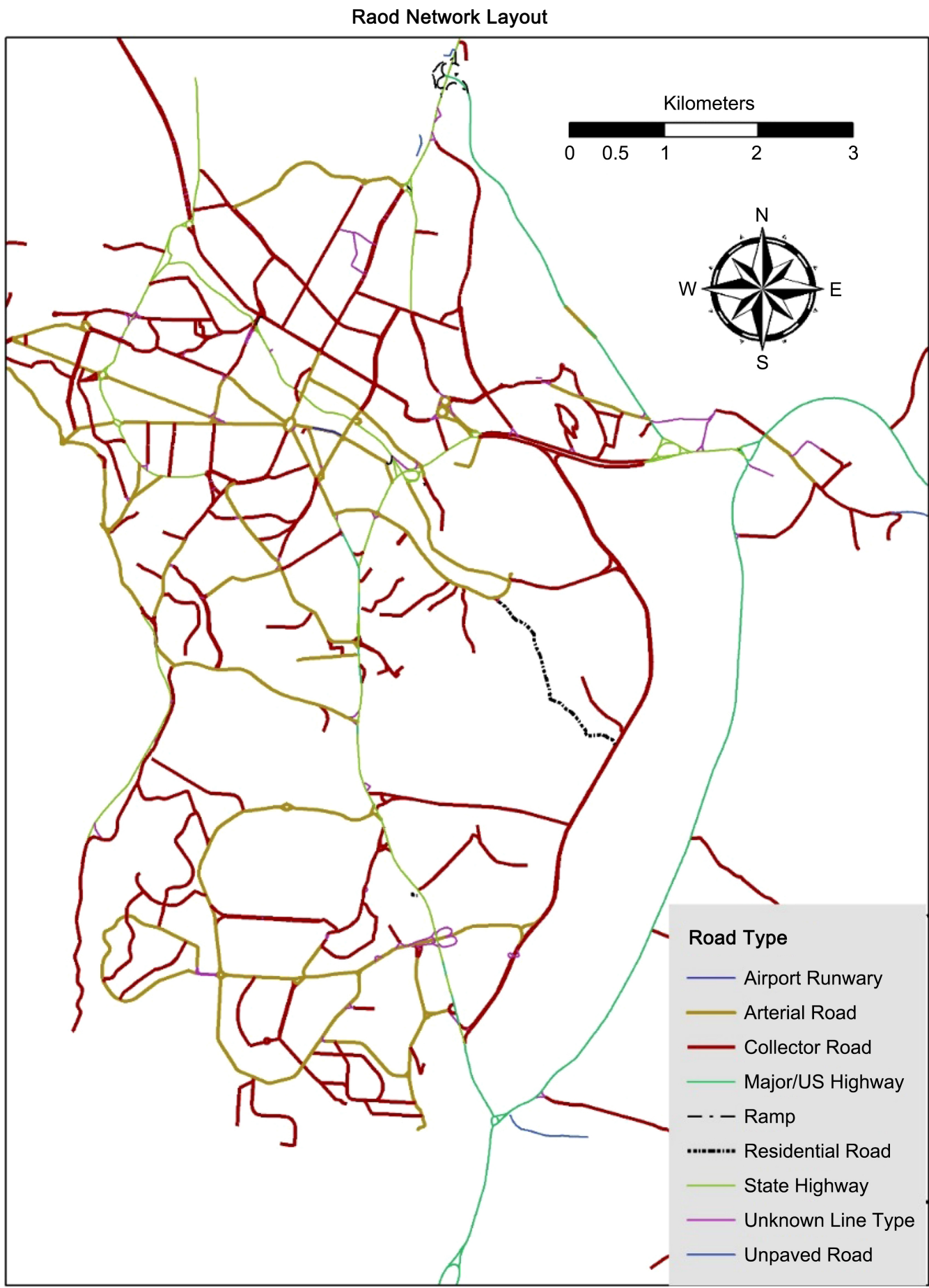

Figure 1. Street network of Sanandaj. 


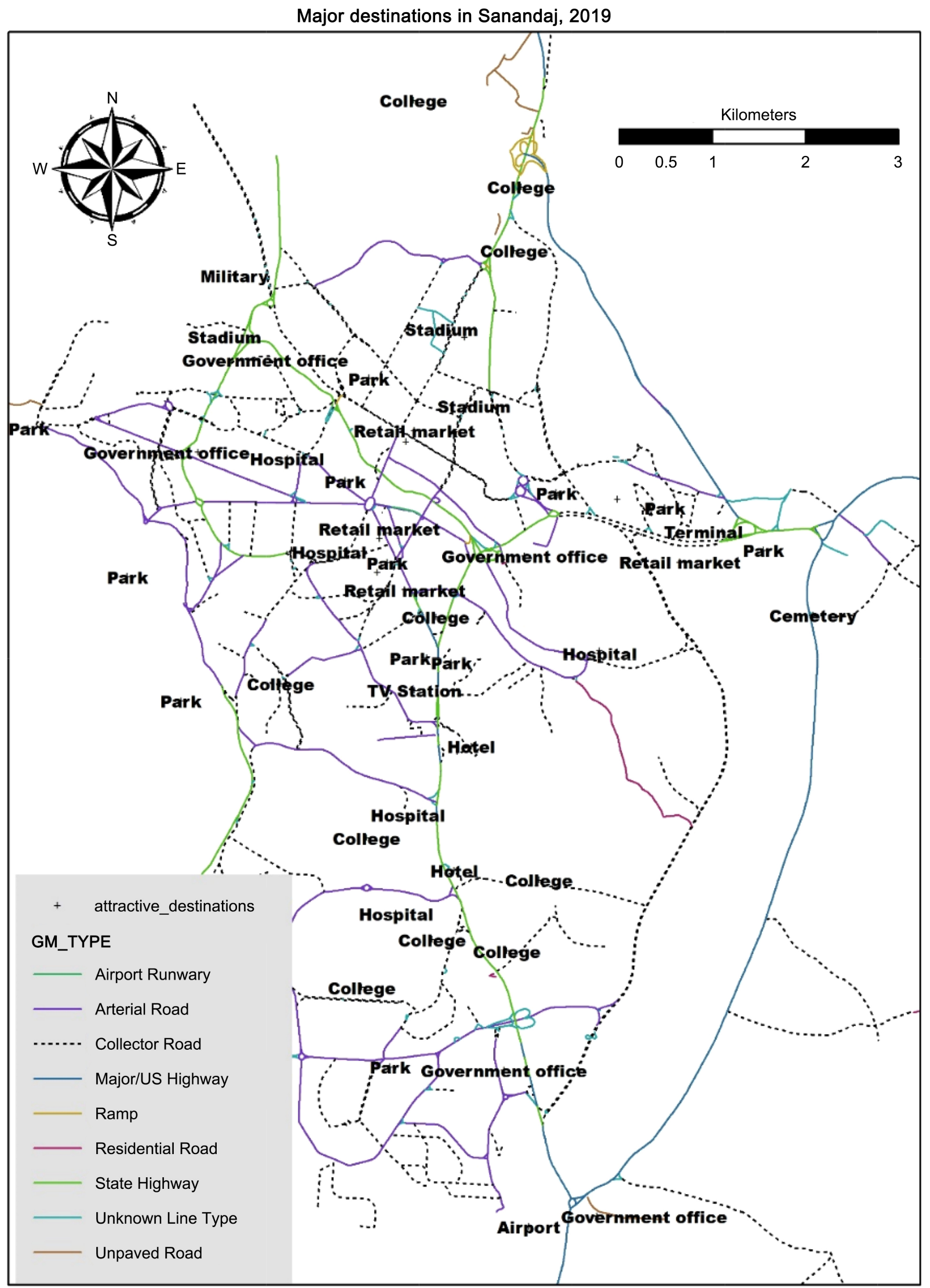

Figure 2. Location of major destinations in Sanandaj. 


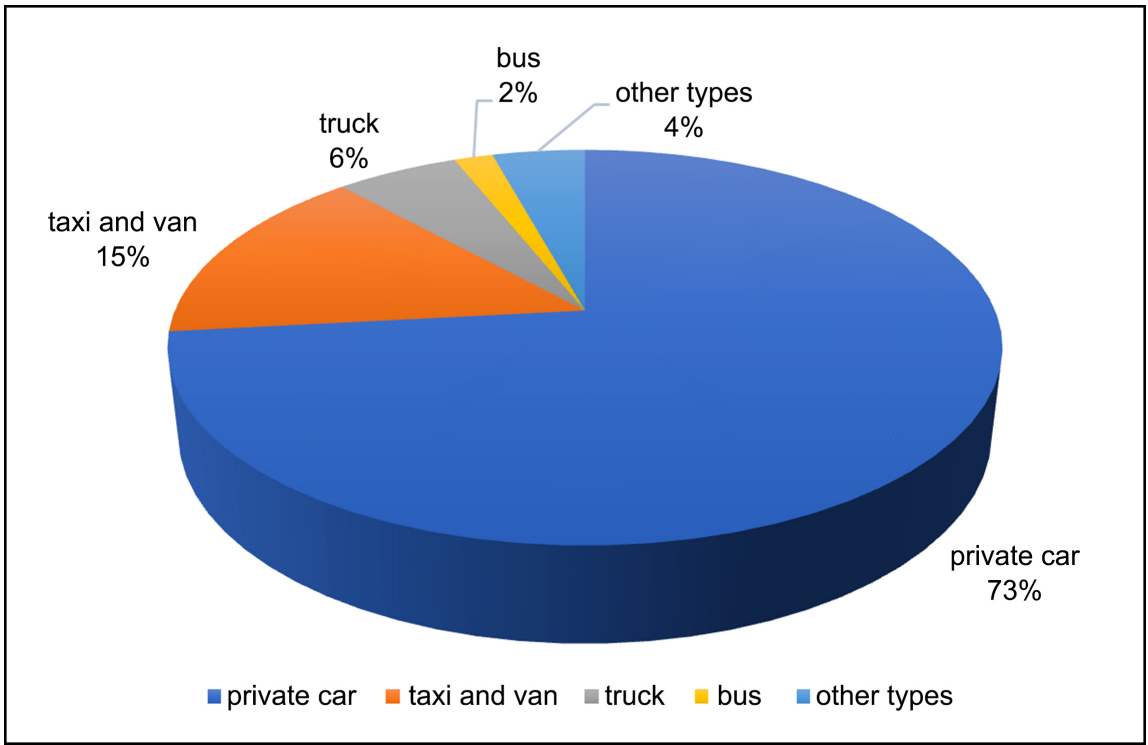

Figure 3. Percentage Share of Transportation Modes in Sanandaj [1].

\section{Methodolgy}

In this section a combination of GIS tools and Honeybee algorithm is used to design the routes for each bus network scenario and assess the results which are the proposed transit network designs. The bus networks provide access to the service for attractive destinations and major population centers in Sanandaj. In the first step of the design GIS is used to draw the bus lines according to the street network and location of major destination and census blocks. In the next step the bus routes are connected to form a bus network. Later, the coverage analysis is performed by GIS in order to determine the accessibility of the proposed designs. Moreover, honeybee algorithm evaluates the designs based on the network design objectives, number of stops and total travel distance on the network, and the results of the evaluation will be analyzed and displayed in several charts in the next section.

\subsection{Honeybee Algorithm}

Karaboga [7] proposed a swarm intelligence method based on the honeybee's search for food in nature and information exchange between bees for minimal forage selection that leads to a collective behavior of the swarm. The model consists of several components: food source, employed forager (i.e., recruiter bee) and unemployed forager (i.e., uncommitted bee), and the model that determines the two behavior modes.

Honeybee optimization methods are based on the concept of a population of agents searching to find the optimal solution. The methods aim at generating a solution set in a predetermined number of iterations and search for the best solution is terminated when certain criteria are satisfied [8]. Honeybees use two operations to find the best solution: forward pass and backward pass [9] [10].

In the first step of this method bees search the solution space with a prede- 
fined number of moves and construct or improve a partial path (i.e. forward pass); afterwards they go back to the hive (i.e., backward pass). In the second step, bees interact and exchange information to know each other's objective function values. With a probability, each bee either picks a new solution and discards its uncompleted solution to be an uncommitted follower of other bees, or dances to recruit others to follow its partial solution. Followers use the partial solution as a base to build their path from its end point [8] [10]. Two phases of forward pass and backward pass are performed several times to provide a complete solution and the algorithm continues to operate until a stopping condition such as predefined number of iterations, or iterations with no improvement in the value of objective function is satisfied.

\subsection{TND Model}

Modelling TND problem involves a process of searching for efficient transit routes based on the preferences of transportation firms and users [4] [11] [12] [13] [14]. In the multi-objective network design, one would define several objectives in order to operationalize the operator's and user's preferences. A weight is assigned to each objective that represents the importance of the objective for the decision maker. Each decision maker, based on his/her preference, assigns different values to objectives' weights. After weights have been determined, a distinct solution is generated. Solutions generated in this way by experts and stakeholders create a set of non-dominated or Pareto optimal solutions that construct a Pareto frontier in the solution space (see section 3.1). This study focuses on following objectives:

1) Number of stops in the transit network.

2) Walking distance to the transit stop.

3) Travel distance of passengers in the transit system.

First objective is based on the operator's perspective to minimize the number of stops in the transit network. The objective objective's formulation is as follows:

$$
f_{2}\left(N_{r n}\right)=\sum_{r=1}^{k} \sum_{n=1}^{m} N_{r n}
$$

where $N_{r n}$ is a decision variable, and $N_{r n}=1$ if a stop $n$ is located on the $r$-th route, otherwise $N_{r n}=0 . \mathrm{n}$ is the stop number and $k$ are the route number. The objective function is subject to some constraints. In order to limit the minimum and maximum number of stops, a distance constraint between two stops should be considered. The minimum and maximum distances between two stops is set to 300 and 600 meters, respectively; these are based on the observed pattern of stops of radial transit networks [15]. Also, the number of stops on each bus route should be greater than zero. The objective function takes the following form:

$$
f_{2}\left(W D_{r n}\right)=\sum_{r=1}^{k} \sum_{n=1}^{m} W D_{r n}
$$

where $W D_{r n}$ is a decision variable of walking distance to the stop $n$ on the $r$-th 
route, $0 \leq W D_{r n} \leq 300$ meters. In order to minimize the total travel distance or time of passengers in the transit system (i.e., the total cost for user) another objective is proposed. The objective is to minimize the difference of travel time between two stops and the shortest path time. The objective function is expressed as follows:

$$
f_{3}\left(t_{r n l}\right)=\sum_{r=1}^{k} \sum_{n=1}^{m} \sum_{l=1}^{o} d_{r n l}\left(t d_{r n l}-s p d_{r n l}\right)
$$

where $t_{r n l}$ is the difference between $t t_{r n l}$ (travel time between stops $I$ and $n$ on the $r$-th route) and $s p t_{r n l}$ (the shortest path between stops $l$ and $n$ on the $r$-th route) [4]. The travel time can be the shortest path time, but in most of the transit networks it is longer than shortest path time in order to provide better coverage for remote areas in the city. $d_{r n l}$ is the travel demand between stops $l$ and $n$ on the $r$-th route; travel demand is constant in this study. Because the real-time data of traffic is not available, the demand is considered based on the population of the census blocks in origin.

\section{Results}

Geographic datasets that are used in the analysis here include ArcGIS shapefiles of the road network, attractive destinations, and census blocks. Road network of Sanandaj consists of different classes of paved roads as major highway, arterial streets, collector streets and residential roads and alleys. A limited number of highways are in Sanandaj which mostly consist of beltways around the city.

The data used to design the transit network include the road network shapefile in GIS, georeferenced census blocks and the census attribute data, attractive destinations, location of peripheral towns. Using the available data, several scenarios are generated in order to be further investigated. Each TND is evaluated based on the objectives proposed such as minimizing walking distance, travel distance and the number of stops. There is a trade-off between minimizing costs and increasing the access to the transit service. Minimizing operational cost can decrease the accessibility, while, increasing the access to transit system is costly, due to the need for bigger fleet and number of routes.

\subsection{Proposed Transit Network Designs (TNDs)}

The proposed TNDs have been displayed in Figure 4 to Figure 14. The networks proposed, except for the first network, include beltways to increase the coverage of system compared to the current bus system, because there are no beltways in the existing bus system of Sanandaj [1]. In the case of Sanandaj, most people travel to CBD and beltway bus lines act as feeder lines between the radial

\subsection{Coverage Analysis of Proposed TNDs}

Increasing the coverage of the bus system for the census blocks and offering the bus service in the walking distance of the blocks is an objective of the transit network design in this study. Using the tools available in GIS, the coverage 
in different neighborhoods of the city and peripheries has been analyzed. The coverage analysis is a two-step approach. In the first step 100-meters and 300 -meters buffer zones around the proposed networks are generated. In the second step, the blocks centroids inside the buffer zones are considered as the population within the walking distanceof the network.

Different minimum and maximum walking distance constraints have been proposed in the literature. The examples are maximum distance between 300 and 400 meters by Alterkawi [16] and 400 meters by Demetsky and Lin [17]. Farwell and Marx [18] suggest that walking distance more than 400 meters is very inconvenient for transit users. Therefore, study the range of possible values for the walking distance to the stops in this research is limited to 300 meters, due to compact urban structure of the study area [1].

Figure 15 displays the population covered for the 11 transit networks proposed for two walking distance scenarios to and from the bus network. Also Figure 15 displays the number of major destinations such as Bazaar, cinemas, hospitals which have been covered by the 11 transit network designs proposed.

The $6^{\text {th }}$ and the $5^{\text {th }}$ networks produce relatively similar coverage for 100-meters and 300-meters walking distances. Considering the two walking distance scenarios, $5^{\text {th }}$ and $6^{\text {th }}$ network designs provide better coverage for the transit system in Sanandaj, compared to other designs. The $11^{\text {th }}$ scenario offers lower access to the major destinations in the city, due to dispersed routes and lack of enough bus lines passing through the downtown core.

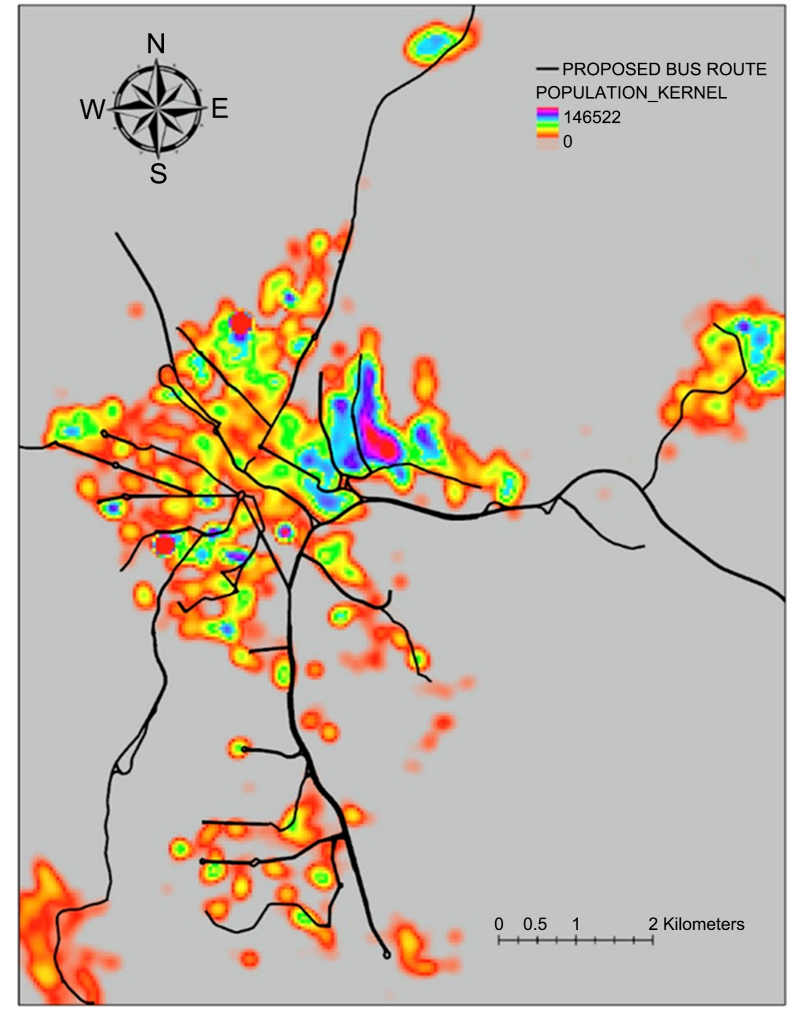

Figure 4. Map of the first proposed bus network and population density of Sanandaj [1]. 


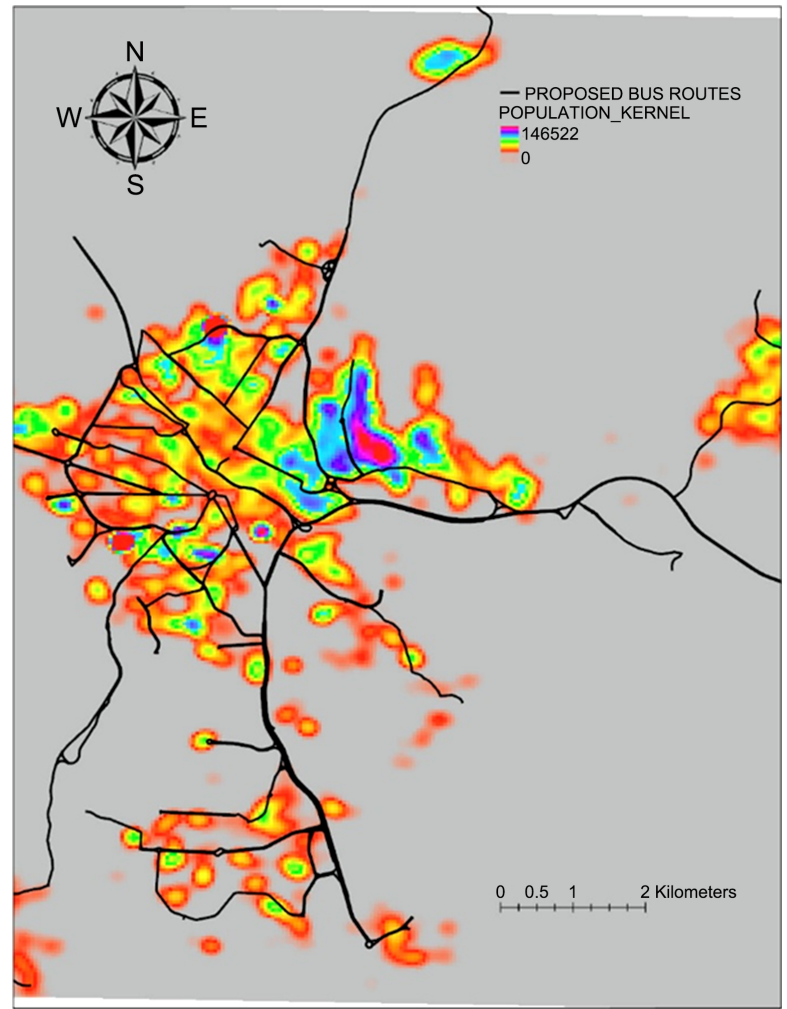

Figure 5. Map of the second proposed bus network and Kernel population density of Sanandaj [1].

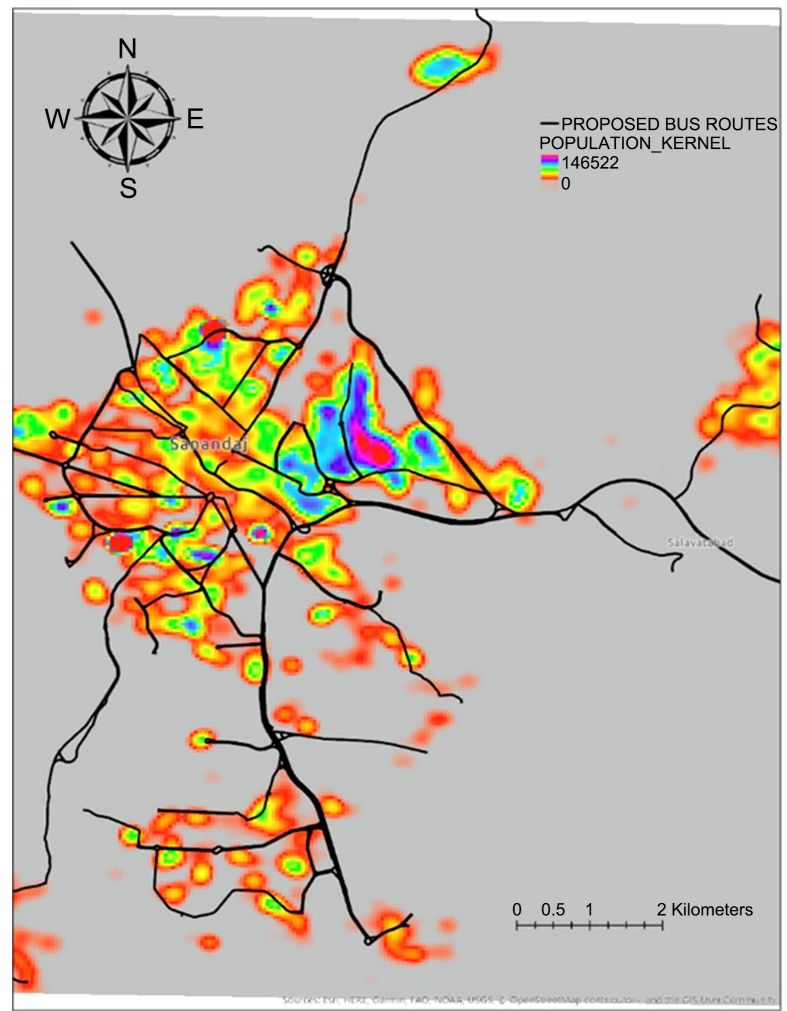

Figure 6. Map of the $3^{\text {rd }}$ proposed bus network and Kernel population density of Sanandaj [1]. 


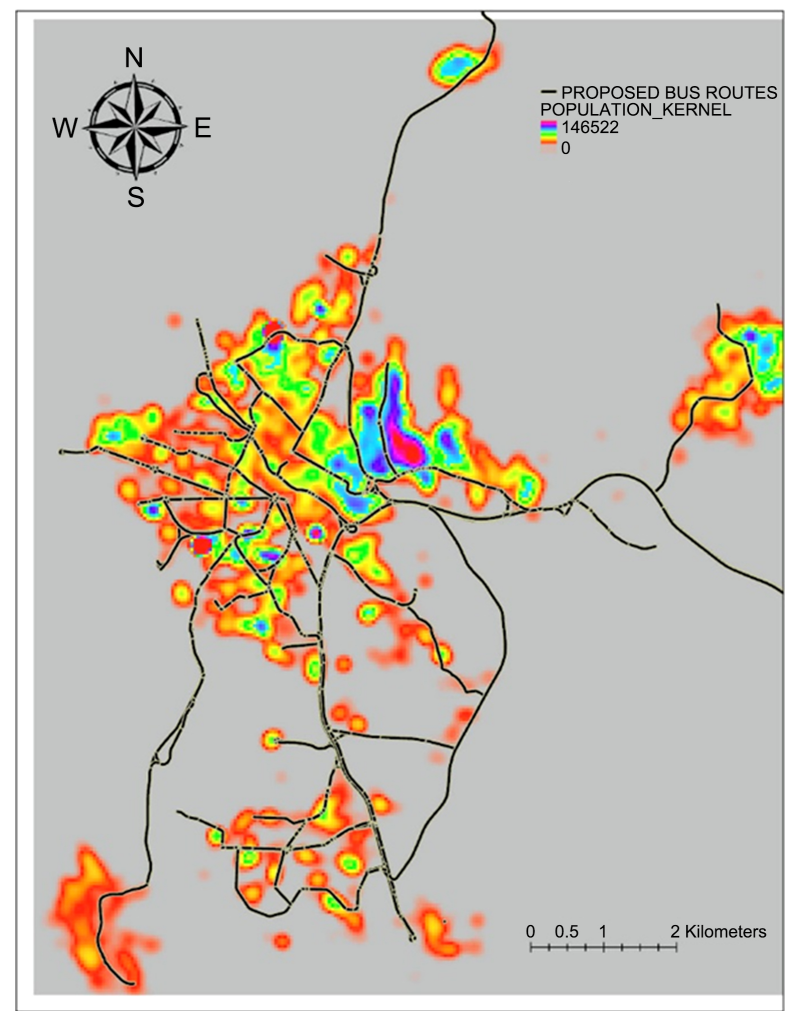

Figure 7. Map of the $4^{\text {th }}$ proposed bus network and Kernel population density of Sanandaj [1].

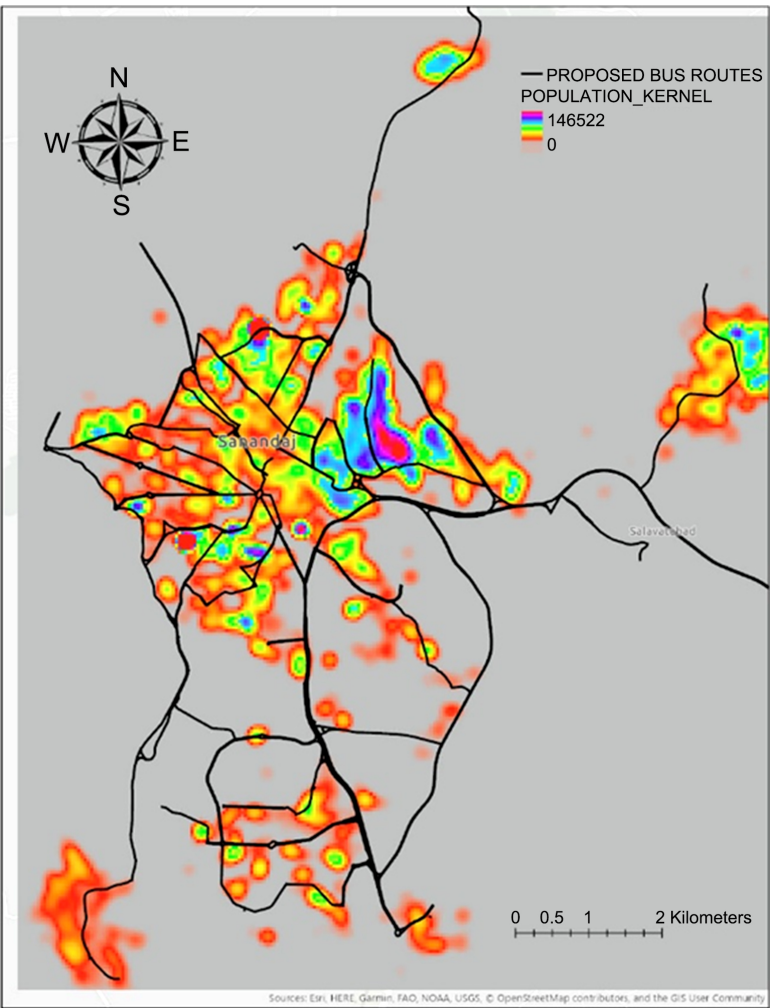

Figure 8. Map of the $5^{\text {th }}$ proposed bus network and Kernel population density of Sanandaj [1]. 


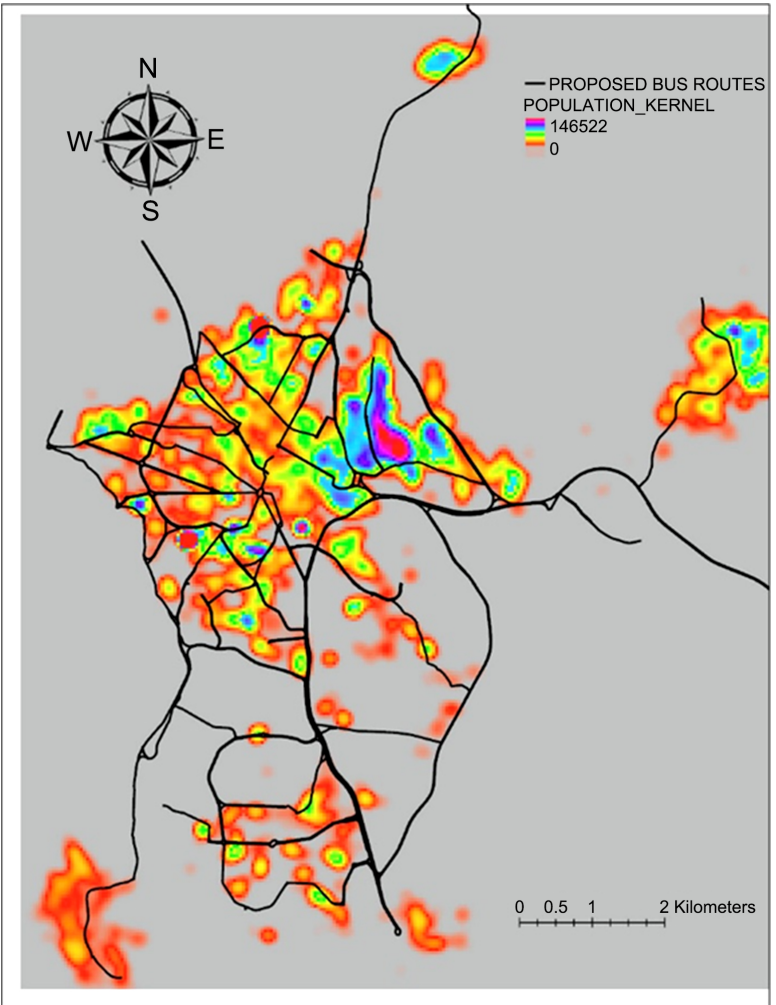

Figure 9. Map of the $6^{\text {th }}$ proposed bus network and Kernel population density of Sanandaj [1].

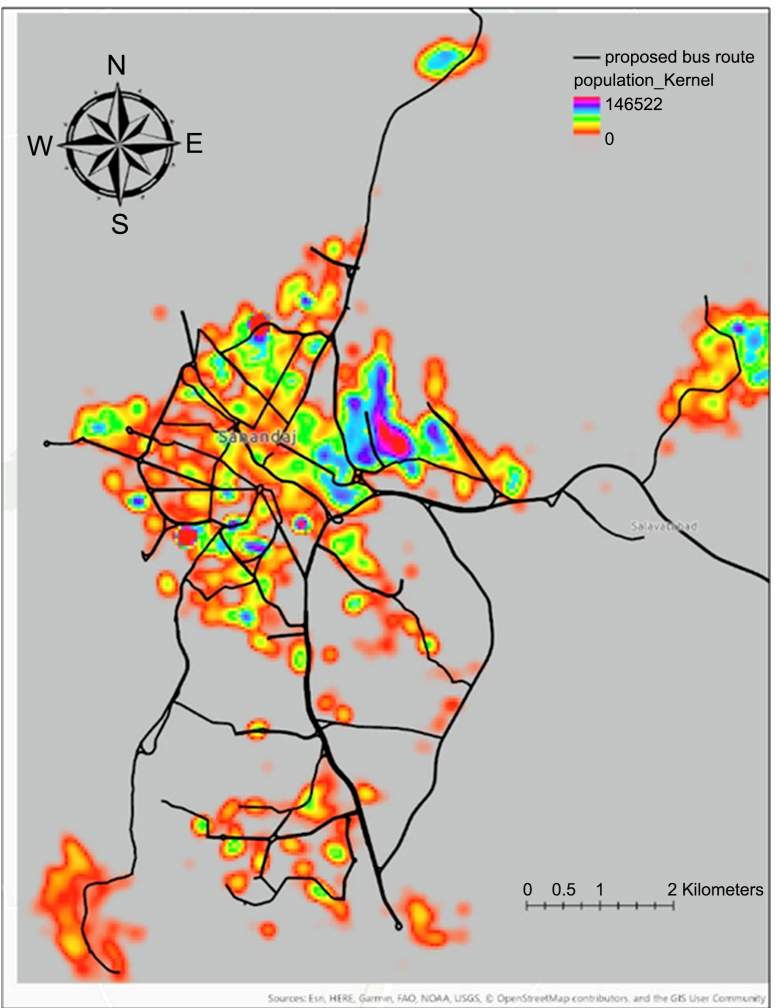

Figure 10. Map of the $7^{\text {th }}$ proposed bus network and Kernel population density of Sanandaj [1]. 


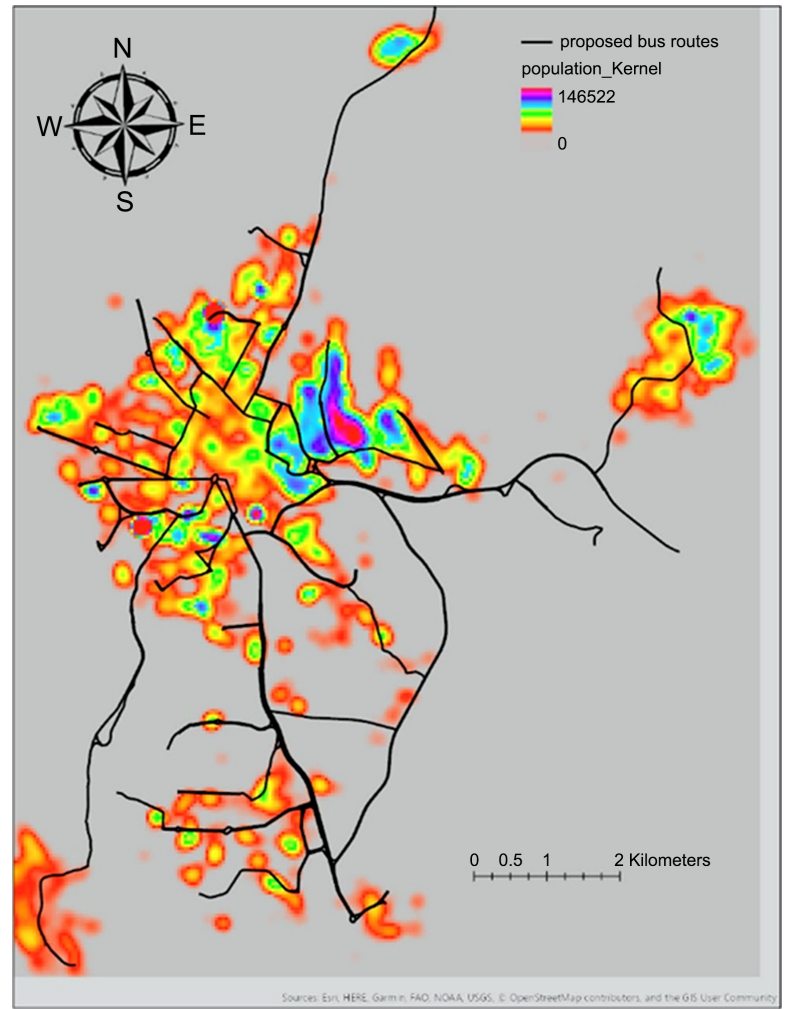

Figure 11. Map of the $8^{\text {th }}$ proposed bus network and Kernel population density of Sanandaj [1].

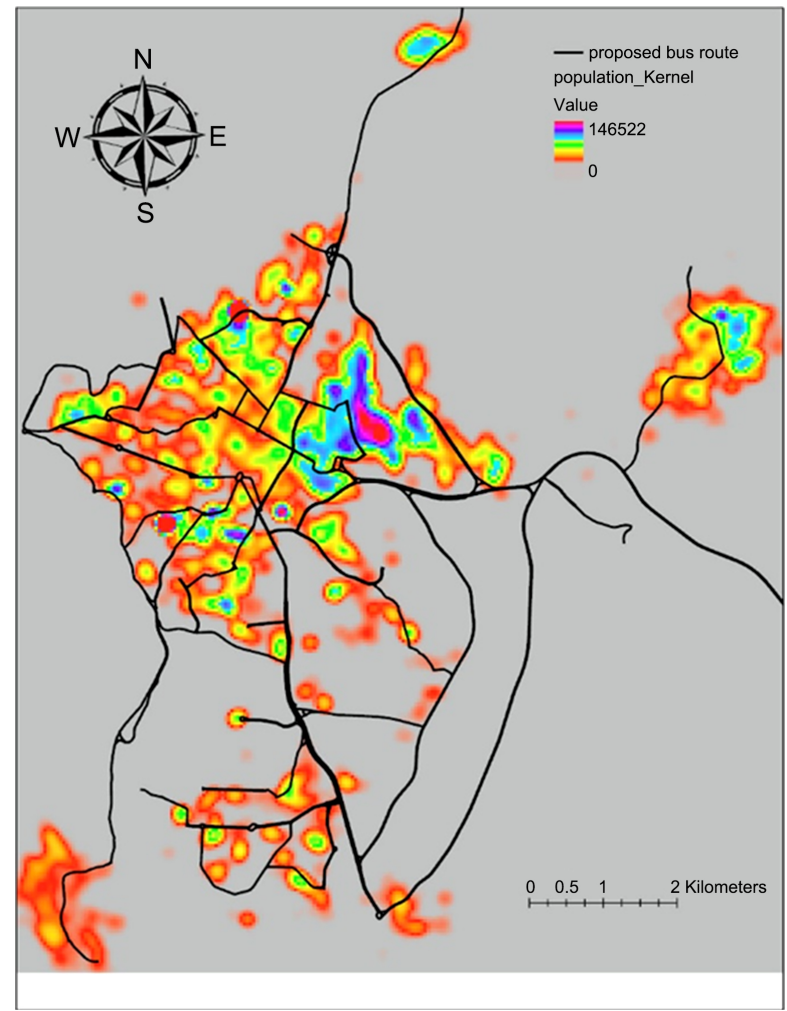

Figure 12. Map of the $9^{\text {th }}$ proposed bus network and Kernel population density of Sanandaj [1]. 


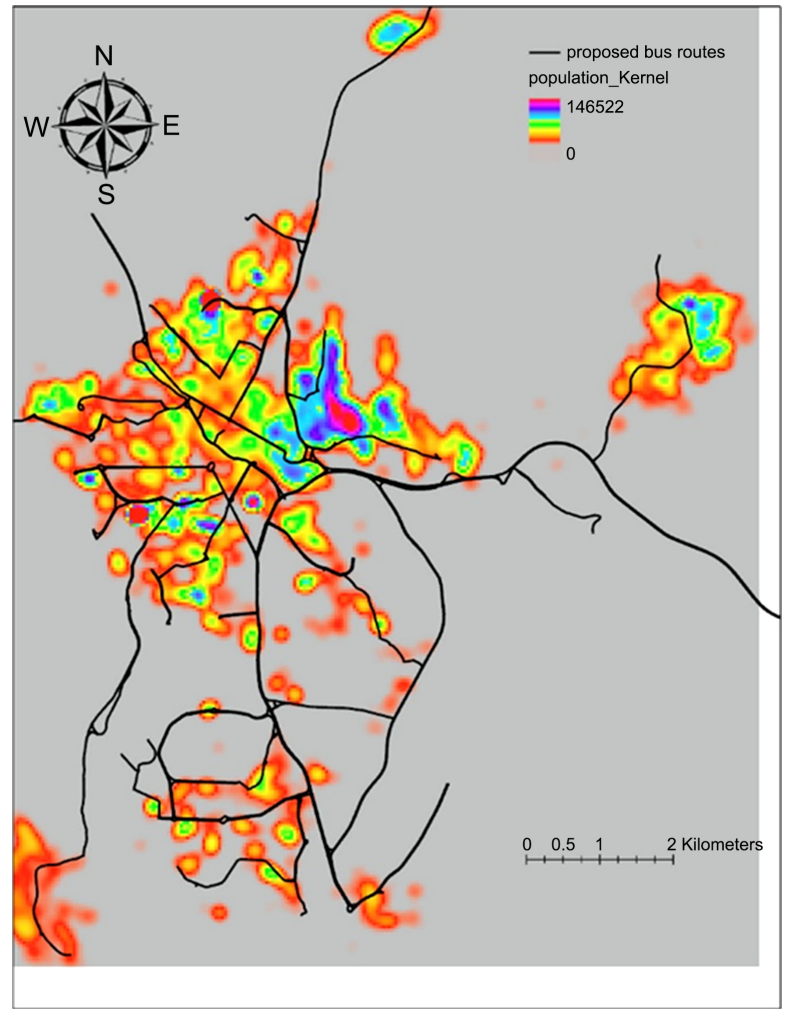

Figure 13. Map of the $10^{\text {th }}$ proposed bus network and Kernel population density of Sanandaj [1].

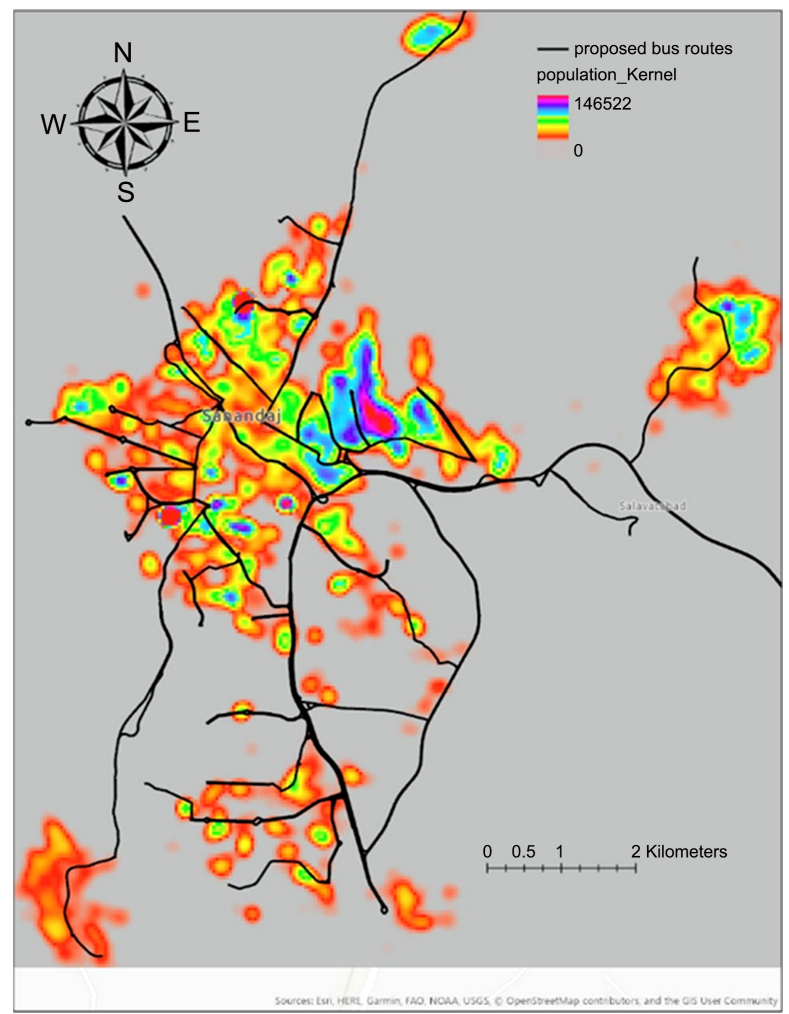

Figure 14. Map of the $11^{\text {th }}$ proposed bus network and Kernel population density of Sanandaj [1]. 


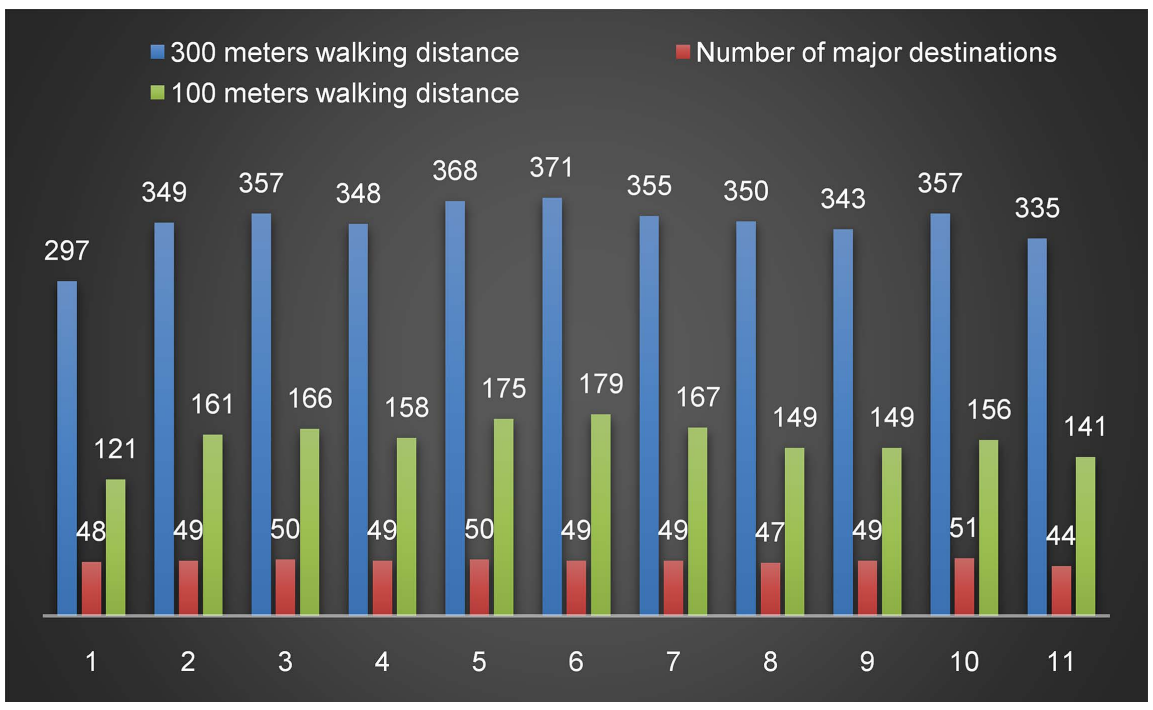

Figure 15. Coverage of Sanandaj population in thousands proposed networks for walking distance of 100 and 300 meters and major destinations.

\subsection{Evaluation of TNDs Using Honeybee Optimization}

The goal of this study is to find bus networks with relatively low travel distance and number of stops with high coverage of the census blocks in Sanandaj. The honeybee algorithm generates a final value for each objective, number of stops and travel distance, for the proposed network designs. In Figure 16, the honeybee evaluation of the transit networks is displayed. The total number of stops and total travel distance are estimated by the honeybee algorithm. The $8^{\text {th }}$ and $10^{\text {th }}$ networks have the lowest numbers of stops among the proposed scenarios. Also, the $1^{\text {st }}$ and the $9^{\text {th }}$ networks have comparatively low number of stops, while the rest of TND scenarios have more than 600 stops, based on the honeybee assessment. Scenarios such as $8^{\text {th }}, 9^{\text {th }}$ and $10^{\text {th }}$ network designs are more suitable options for transit system in Sanandaj based on the three objectives used to evaluate the proposed networks. The existing bus system in Sanandaj has a low performance and it is not considered as an optimal option for the transit system and several bus networks proposed here are more suitable design considering the objectives.

\section{Conclusions and Contribution}

The larger networks proposed in this study provide several beltway lines to cover the peripheral areas and they increase the operation costs, but they also generate higher access to the bus service in the peripheries of Sanandaj. In other words, networks with longer travel distances offer a better coverage of the census block, based on the results.

The current network has a low travel distance, but the major issue is the very low coverage of the existing network [1]. This is due to small network size and lower accessibility of the current bus system in the population centers, which makes it an inconvenient transit system. The evidence is that only the 2 percent 


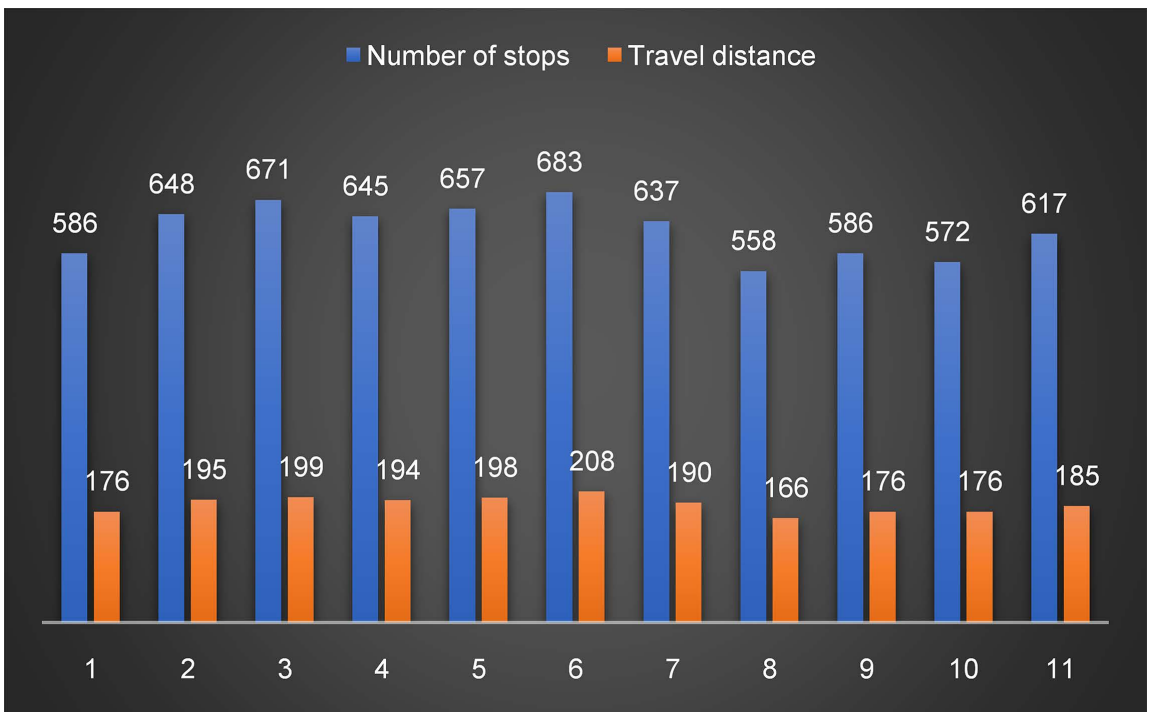

Figure 16. Number of stops generated using Honeybee method for TND scenarios.

of trips made in Sanandaj is by bus [1]. Several scenarios in this study provide a better access to the bus network for the census blocks. One of the limitations of this research is the exact location of stops that are not considered in the analysis and changing the location of stops can change the coverage of the scenarios, to some extent.

Radial designs such as the first scenario are more favorable for operators because of lower operation costs, but they also reduce the level of service in the peripheries. Therefore, a radial network is not favorable for the users of the bus system. Other networks with more beltways are more costly to operate, but they provide better access to the transit system for the population in Sanandaj. The choice for Sanandaj depends on the budgetary restrictions of the city administration, because adding beltways to the bus system generates better access but it also increases the cost of the operation.

This research provides a new approach to transit network design by offering a procedure that combines the abilities of GIS and Honeybee algorithm in the design process. The steps to design and evaluate the bus networks in the procedure can provide better insight about how to design the bus system for future research. Also, for future studies, using detailed information of census data and surveys to further investigate the impact of walking and travel distance on the choice of travel mode, is recommended.

\section{Conflicts of Interest}

The author declares no conflicts of interest regarding the publication of this paper.

\section{References}

[1] Local Government of Kurdistan (2011) Comprehensive Traffic Plan. Local Government of Kurdistan, Sanandaj, 1-200. 
[2] Fan, L. and Mumford, C.L. (2010) A Metaheuristic Approach to the Urban Transit Routing Problem. Journal of Heuristics, 16, 353-372. https://doi.org/10.1007/s10732-008-9089-8

[3] Zhu, Z., et al. (2017) Route Design Model of Feeder Bus Service for Urban Rail Transit Stations. Mathematical Problems in Engineering, 2017, Article ID: 1090457. https://doi.org/10.1155/2017/1090457

[4] Ceder, A. (2016) Public Transit Planning and Operation: Modeling, Practice and Behavior. CRC Press, Abingdon. https://doi.org/10.1201/b18689

[5] Murray, A.T. and Wu, X. (2003) Accessibility Tradeoffs in Public Transit Planning. Journal of Geographical Systems, 5, 93-107. https://doi.org/10.1007/s101090300105

[6] Liu, S. and Zhu, X. (2004) Accessibility Analyst: An Integrated GIS Tool for Accessibility Analysis in Urban Transportation Planning. Environment and Planning B: Planning and Design, 31, 105-124. https://doi.org/10.1068/b305

[7] Karaboga, D. (2005) An Idea Based on Honey Bee Swarm for Numerical Optimization. Erciyes University, Engineering Faculty, Computer Engineering Department, Kayseri.

[8] Nikolić, M. and Teodorović, D. (2013) Transit Network Design by Bee Colony Optimization. Expert Systems with Applications, 40, 5945-5955. https://doi.org/10.1016/j.eswa.2013.05.002

[9] Yang, X.-S., et al. (2013) Swarm Intelligence and Bio-Inspired Computation: Theory and Applications. Newnes, Burlington. https://doi.org/10.1016/B978-0-12-405163-8.00001-6

[10] Nikolić, M. and Teodorović, D. (2013) Empirical Study of the Bee Colony Optimization (BCO) Algorithm. Expert Systems with Applications, 40, 4609-4620. https://doi.org/10.1016/j.eswa.2013.01.063

[11] Petrelli, M. (2004) A Transit Network Design Model for Urban Areas. WIT Transactions on the Built Environment, 75, 1-100.

[12] Ceder, A. and Israeli, Y. (1998) User and Operator Perspectives in Transit Network Design. Transportation Research Record: Journal of the Transportation Research Board, 1623, 3-7. https://doi.org/10.3141/1623-01

[13] Bagloee, S.A. and Ceder, A.A. (2011) Transit-Network Design Methodology for Actual-Size Road Networks. Transportation Research Part B: Methodological, 45, 1787-1804. https://doi.org/10.1016/j.trb.2011.07.005

[14] Fusco, G., Gori, S. and Petrelli, M. (2002) A Heuristic Transit Network Design Algorithm for Medium Size Towns. Proceedings of the 13th Mini-EURO Conference, Bari.

[15] Badia, H., Estrada, M. and Robusté, F. (2014) Competitive Transit Network Design in Cities with Radial Street Patterns. Transportation Research Part B: Methodological, 59, 161-181. https://doi.org/10.1016/j.trb.2013.11.006

[16] Alterkawi, M.M. (2006) A Computer Simulation Analysis for Optimizing Bus Stops Spacing: The Case of Riyadh, Saudi Arabia. Habitat International, 30, 500-508. https://doi.org/10.1016/j.habitatint.2004.12.005

[17] Demetsky, M.J. and Lin, B.B.-M. (1982) Bus Stop Location and Design. Journal of Transportation Engineering, 108, 313-327.

[18] Farwell, R. and Marx, E. (1996) Planning, Implementation, and Evaluation of OmniRide Demand-Driven Transit Operations: Feeder and Flex-Route Services. Transportation Research Record: Journal of the Transportation Research Board, 1557, 1-9. https://doi.org/10.1177/0361198196155700101 